\title{
Drawbacks of Poor-Quality Ultrasound Images and its Enhancement
}

\author{
Khan Sumaiya \\ Department of Computer Science and Information \\ Technology \\ Dr. Babasaheb Ambedkar Marathwada University, \\ Aurangabad-431001, Maharashtra, India
}

\author{
S.S. Kawathekar, $\mathrm{PhD}$ \\ Department of Computer Science and Information \\ Technology \\ Dr. Babasaheb Ambedkar Marathwada University, \\ Aurangabad-431001, \\ Maharashtra, India
}

\begin{abstract}
Ultrasound waves are widely used in the field of medical sciences for diagnosis, therapeutic, and interventional use. It is commonly used in the areas of cardiology, urology, general abdominal imaging, obstetrics and gynecology, vascular imaging, and as a guide in surgical procedures. The noninvasive, cost-effective, flexible, and radiation-free properties of ultrasonography have made it popular among healthcare professionals. The ultrasound images are generated through pulsed acoustic waves that are transmitted and received by a transducer in the ultrasound machine. However, the quality of an image is affected when ultrasound signals degrade while propagating through biological tissues. The poor image quality affects the interpretation, thereby, delaying the diagnosis and intervention in the patient. The present article discusses the basics of ultrasonography, highlights various artifacts in an ultrasound image, and reviews some methods for the enhancement of ultrasound images.
\end{abstract}

\section{General Terms}

Image enhancement

\section{Keywords}

Ultrasound imaging, Ultrasound artifacts, Ultrasound enhancement, Ultrasound basics

\section{INTRODUCTION}

Ultrasound imaging is widely used in medical sciences due to its popularity, versatility, and affordability $[1,2,3]$.
Ultrasonography is a non-invasive technique that delivers a cross-sectional examination of human anatomical structures in the field of cardiology, gastroenterology, obstetrics and gynecology, and vascular imaging. Additionally, it is used as a guide in surgical procedures [4]. Ultrasonography scanners generate the images by using pulsed acoustic waves at a frequency ranging between 1-20 MHz. These waves that are transmitted through and reflected by the tissues are received by the transducer, thereby, forming an image of the organ [5]. This detailed principle of echo imaging is discussed below in the paper.

The development of ultrasonography dates back to the late 1700s when Lazzaro Spallanzani hypothesized that bats used sound to navigate. Later in 1842, Christian Doppler first postulated the Doppler Effect that forms the basis of ultrasonographic evaluation of blood flow. In 1877, Jacques and Pierre Curie discovered piezoelectricity, which formed the basis for the development of ultrasonography transducers. In the twentieth century, ultrasonography was gaining popularity as a therapeutic tool in the field of rheumatology, neurosurgery, and rehabilitation. In 1942, medical ultrasonography was first used by Karl Theo Dussik, a neurologist, to depict changes in the size of the brain ventricle secondary to tumor growth [Figure 1]. Over the years, ultrasound imaging techniques have evolved to such an extent that these machines are providing innovative ways to improve diagnostic, therapeutic, and interventional aspects of ultrasonography [6].

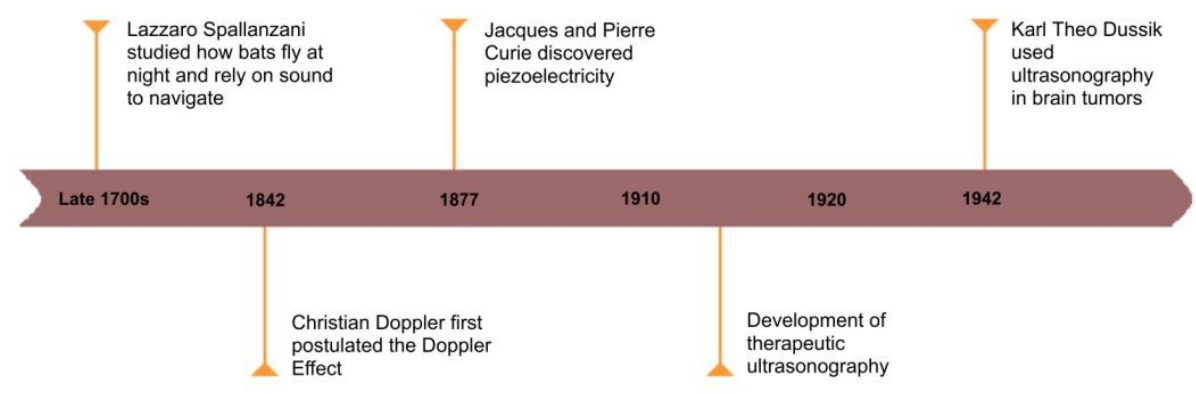

Figure 1: Developmental history of ultrasonography

Therefore, since its inception, ultrasonography has gained popularity as it is an inexpensive, non-invasive technique, compact and portable, and provides real-time images. Furthermore, no ionizing radiations are emitted, thereby, restricting the need for separate environments like radiationshielded walls $[7,8,9]$.
Nevertheless, ultrasonography has several drawbacks, mainly of limited penetration through bone or gas-filled structures, thereby, restricting its use in the brain, lungs, and the abdominal region [10]. Moreover, ultrasound image quality is another major concern as poor quality images limit its readability and interpretation to obtain quantitative 
information [2]. The present review article focuses on the various artifacts that affect the quality of an ultrasound image and methods to enhance them.

\section{CONVERTING SOUND TO IMAGE: PHYSICS BEHIND \\ ULTRASONOGRAPHY}

Ultrasound is made up of mechanical waves comprising of a frequency greater than $20 \mathrm{kHz}$ (the upper human auditory limit). Medical ultrasound machines use these waves with a frequency ranging between 2 and $15 \mathrm{MHz}[11,12]$. The production of these waves depends on a phenomenon called the piezoelectric effect. This effect is exhibited by some crystalline materials, called a piezoelectric crystal that reversibly converts mechanical and electrical energies. When these crystals are compressed or stretched, mechanical energy converts to electrical energy, thereby, generating electrical energy on their surfaces. This is called the piezoelectric effect. However, these crystals generate a reverse piezoelectric effect by expanding or contracting after a potential difference is applied between the faces of a piezoelectric crystal. This results in the conversion of electrical energy to mechanical energy [13]. This phenomenon is depicted below [Figure 2].

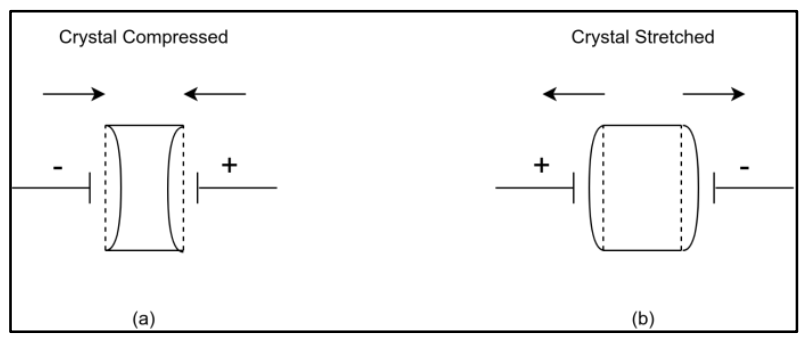

Figure 2: (a) Piezoelectric effect; (b) Reverse piezoelectric effect

In a clinical ultrasound machine, the transducer consists of the tiny piezoelectric crystals that generate multiple ultrasound waves [Figure 3]. When an alternating current is transmitted through these crystals, they vibrate at a high frequency and produce an ultrasound beam that traverses through the body tissues with the aid of a conductive gel. The beam passes through various body tissues underneath and returns to the transducer resulting in a shape change of the crystals. This minor change generates an electric current that develops into an ultrasound image after amplification $[4,11]$.

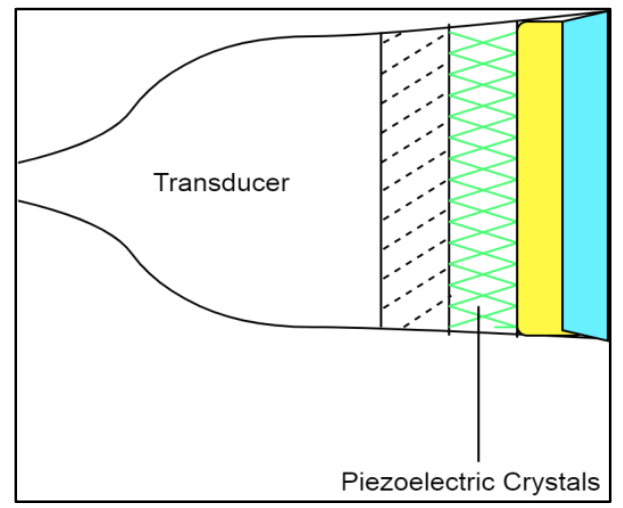

Figure 3: Piezoelectric crystals in a transducer

Furthermore, when these ultrasound beams travel through the tissues, part of the energy is reflected and part is transmitted. The tendency to resist the movement of ultrasound through the tissues is called acoustic impedance [14]. The resistance depends on the density of the substance through which the ultrasound passes. The greater the density of the tissues more will be the reflection of the ultrasound beams [Figure 4] [15]. Therefore, denser tissues, such as bones reflect more energy towards the probe and produce white bright images or hyperechoic images, while the poor reflector like plasma fluids or blood will produce a black or hypoechoic image [11, $14,16,17]$.

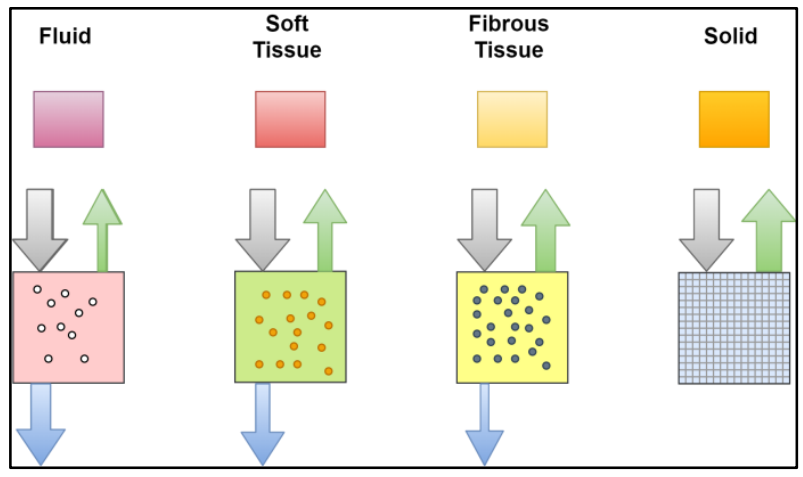

Figure 4: Acoustic impedance of various tissues based on density

\section{BASIC EQUIPMENT OF ULTRASOUND MACHINE}

The ultrasonography machines are composed of the following components:

\subsection{Transducer probe}

The transducer probe is a part that converts ultrasound beams to an ultrasound image, as discussed earlier. It is comprised of a transducer head, a connecting wire, and a connector that connects it to the ultrasound machine. The transducer's head consists of a footprint region that transmits and receives the ultrasound waves. There are mainly three types of transducers: curvilinear, linear, and sector. The characteristics of waves transmitted by these transducers are depicted in Figure $5[18,19]$.

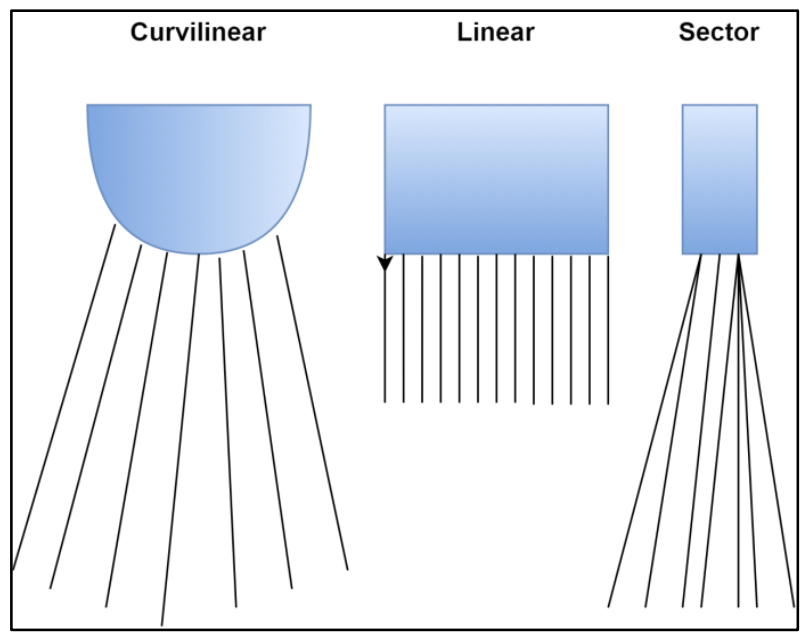

Figure 5: Transmission waves from various types of transducers

Linear transducers produce parallel sound waves along with a rectangular image on the screen. They produce a good near field resolution as the number of scan lines and image width is uniform throughout the tissue levels. However, these cannot be used on the curved body parts due to the formation of air 
gaps between the transducer and the skin. Curvilinear transducers produce curvilinear images at a frequency ranging between 2-7 MHz and with a wide field of view. As the distance of these waves increases from the transducer, the density of scan lines decreases. These transducers are preferred for abdominal scanning due to the curvature of the abdominal wall. Sector transducers are not frequently used in clinical ultrasonography as they produce images with poor near field resolution and are difficult to manipulate. They produce a narrow fan-like image that increases in width with deeper penetration. These are mainly used for scanning small anatomic sites [18, 20,21].

\subsection{The transmitter}

The transmitter is an electronic circuit that applies an electrical modulated pulse to the transducer at 100 nanoseconds. The amplitude of this tension is based on the type of tissue and the penetrating depth $[18,20]$.

\subsection{The receiver}

The receiver is an amplifier that receives signals from the transducer. It has a bandwidth between 1 and $10 \mathrm{MHz}$. It is important to control gain on the amplifier as it manages the attenuation by the depth and other types of internal interfaces [20].

\subsection{Digital Scan Converter}

A digital scan converter converts the received data from an analog to a digital image and stores in the receiver [20].

\subsection{Aids for measurement and register}

The ultrasound machine consists of various accessories such as the keyboards, mouse, printers, and remote handcontrollers to save the operation data or register the images for future analysis [20-22].

\section{ULTRASOUND IMAGE MODES}

Medical imaging utilizes several modes of ultrasound [23], mainly classified as greyscale imaging mode and Doppler imaging mode [19].

\subsection{Greyscale imaging modes}

Greyscale imaging modes are composed of A-mode, B-mode, and M-Mode. A-mode, also known as amplitude mode, is the simplest type of ultrasound mode. It uses a single transducer to scan the body and generates a graph based on the echo and the depth of scanning. The $\mathrm{x}$-axis represents the depth in tissues and the $y$-axis represents the amplitude of the returning wave.

The second mode is B-mode, alternatively known as brightness mode or two-dimensional imaging mode. It is the most commonly used form of greyscale mode. In this mode, a linear array of transducers performs simultaneous scans through the body, thereby, producing two-dimensional ultrasound images. Images in the B-mode are obtained in realtime and depending on the intensity of the returning ultrasound beam [Figure 6].

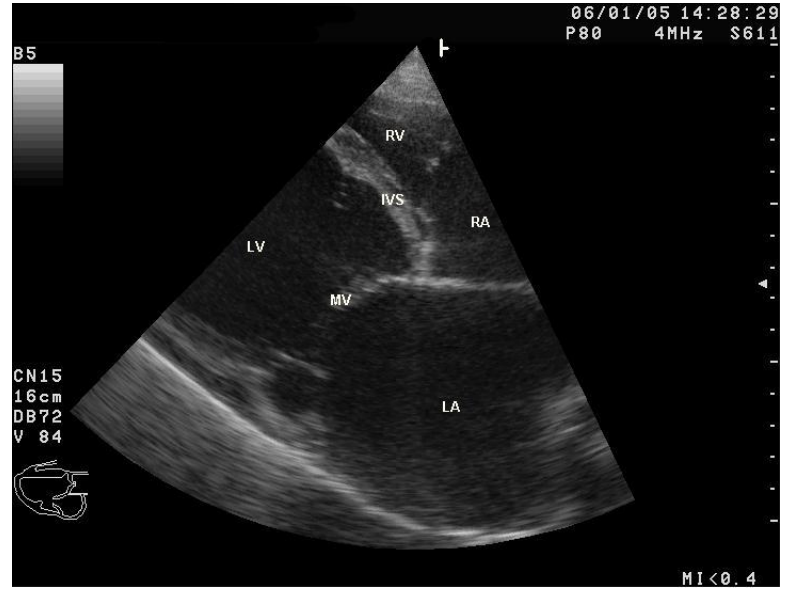

Figure 6: B-mode ultrasound image

M-mode, also known as motion mode, is infrequently used in current ultrasound imaging. It originates from a single beam that penetrates through the organs with a high pulse repetition frequency. It is specifically used to assess certain fetal cardiac conditions [Figure 7] [17, 23, 24].

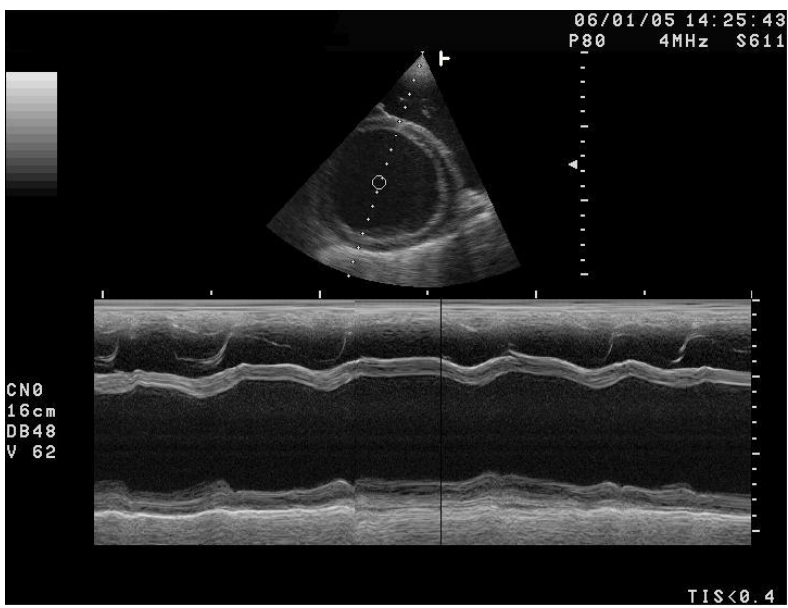

Figure 7: M-mode ultrasound image

\subsection{Doppler imaging mode}

Doppler imaging mode uses the principle of Doppler Effect to visualize and measure the blood flow through vessels [23, 24]. Doppler Effect is described as the apparent variation in the frequency of a sound wave when the source approaches near or moves away from the observer. In clinical practice, when an ultrasound beam insonates a certain blood vessel at a particular frequency, the shift in frequency is directly proportional to the speed of red blood cell movement within the particular blood vessel. This shift in frequency is displayed as a time-dependent plot on the ultrasonography machine [Figure 8] [17, 24] 


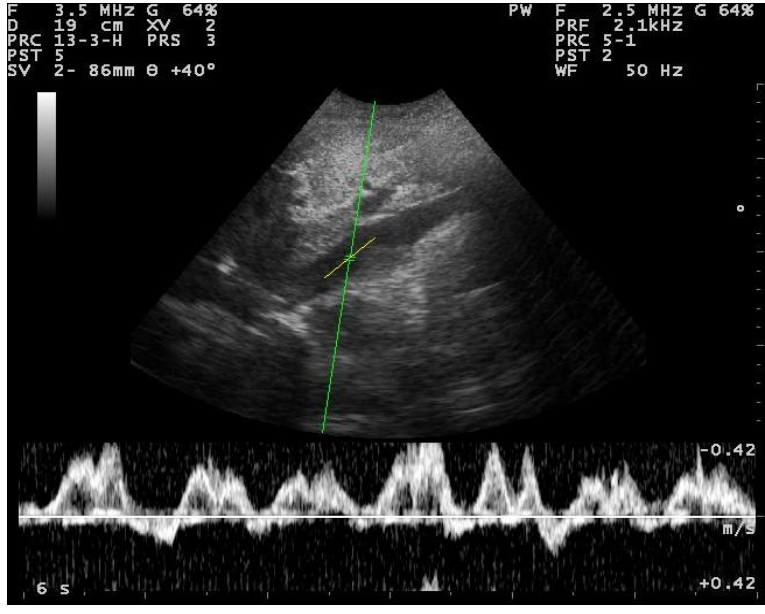

Figure 8: Doppler ultrasound image

Doppler principle is utilized by five types of imaging techniques, as shown below [Figure 9]:

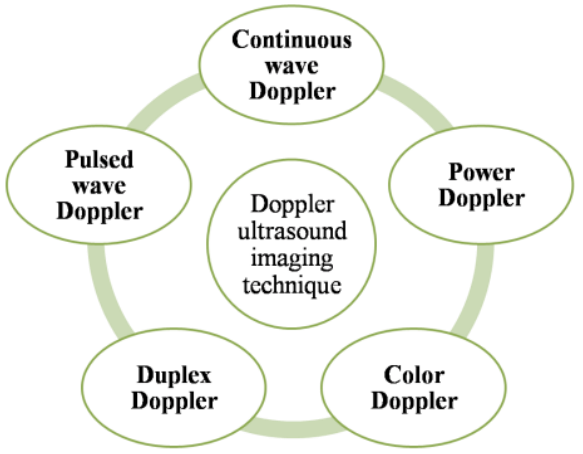

Figure 9: Types of Doppler ultrasound imaging techniques

\subsubsection{Continuous-wave Doppler}

The transducer in the continuous-wave Doppler mode contains two piezoelectric crystals in the same head. One crystal transmits a continuous signal at a frequency of 3-8 $\mathrm{MHz}$, while the other crystal receives the reflected echoes and records its frequency. The difference, also called Doppler shift, usually falls within the audible range. Continuous-wave Doppler is used for the detection of blood flow in vessels; however, it does not provide any information on the depth, velocity, and direction of blood flow [25].

\subsubsection{Pulsed wave Doppler}

Pulsed wave Doppler detects the depth at the origination site of the returning signal. Three technical parameters result in the detection of such mobile structures. These are as follows:

\section{* The high pulse repetition frequency \\ * Low transducer frequency for deeper structures \\ * Insonation angle less than $60^{\circ}$}

Despite the detection of the depth, pulsed wave Doppler fails to provide any information on the structure or site of origin of a reflected echo [25].

\subsubsection{Color Doppler}

Color Doppler is used to detecting the presence of vascular blood flow within the insonated tissue. In this mode, the echo signals/ pulse packets are received through the applied transmit-receive pulse signals along with a series of locations in an ultrasound beamwidth. This data is assigned a red color by the machine if the blood flow is towards the transducer and it shows blue color if the flow is away from the transducer. Furthermore, the image is superimposed on the real-time Bmode image for stationary structures within the beamwidth.

\subsubsection{Power Doppler imaging}

Power Doppler is a sensitive mode of Doppler that helps in the detection of the low-velocity flow of blood. This model estimates the strength/amplitude of the scattered velocities by color Doppler at positions throughout the scanned field. The image generated is continuous and is not affected by the relative flow direction. However, power Doppler does not assess the direction and the velocity of blood flow. Moreover, slow-moving soft tissue signals appear as flash artifacts due to averaging of information at slower frame rates.

\subsubsection{Spectral Doppler}

In this mode, a quantitative assessment of vascular flow is obtained at any point within a blood vessel. Each of the transmitted Doppler signals will have a different phase and amplitude, therefore, the final image developed from the sampled signal will have contributions from all the signals $[17,25]$.

\section{IMAGE CHARACTERISTICS OF AN ULTRASOUND}

Medical ultrasound scanning is widely utilized in the fields of cardiology, anesthesia, critical care, and pain management. It is used typically for the assessment of hemodynamic instability, determination of left ventricular function and cardiac output, assistance with difficult venous access, in addition to facilitation of accurate neural block [26-29]. Therefore, for accurate interpretation of the ultrasound images, the image quality plays an essential role to provide optimum diagnostic and therapeutic intervention. The factors influencing image quality are as follows:

\subsection{Resolution}

Resolution is defined as the ability of an ultrasound machine to distinguish between two objects [30]. There are three types of resolution: spatial resolution, temporal resolution, and contrast resolution [26].

\subsubsection{Spatial resolution}

Spatial resolution is the ability of an ultrasound machine to distinguish between two points at a particular depth in the tissue as determined by the transducer [26]. This is divided into axial and lateral resolution $[24,31]$.

Axial/ longitudinal resolution is the minimum distance between two different structures located at different depths and parallel to the direction of ultrasound [14, 24, 26, 31]. It is roughly equal to one half of the pulse length; therefore, high axial resolution is observed with short spatial pulse length [14, 26]. This indicates that the higher frequency probes produce the best axial resolution; however, there is a high risk of attenuation with high-frequency waves, thereby, resulting in poor penetration through the tissues [14].

Lateral resolution is the minimum distance between two different structures located perpendicular to the direction of the ultrasound beam. Lateral resolution is inversely proportional to the width of the ultrasound beam; therefore, it is high when the width of the beam is narrow $[26,31]$. Similar to axial resolution, narrow beams are generated at high frequencies resulting in poor penetration [24].

\subsubsection{Temporal resolution}

Temporal resolution represents the ability of an ultrasound 
machine to distinguish between the immediate events of rapidly moving structures. The temporal resolution may be improved reducing the depth of penetration, scan lines per frame, and the number of focal points. This means that high temporal resolution is obtained with shallow penetration depth and short pulse repetition period [26].

\subsubsection{Contrast resolution}

Contrast resolution allows an ultrasound system to distinguish between different amplitudes of echo produced by adjacent structures. It may be enhanced by the use of contrast agents, compression, and image memory [26]. The echoes of other tissues obtained through contrast resolution are compared with those from fat. Therefore, the overall gain of the machine should be adjusted so that fat has an isoechoic appearance [31].

\subsection{Image uniformity}

Attenuation is described as a loss of energy when the wave propagates through various tissues that result in poor image quality [19]. The variations of attenuation in ultrasound imaging are compensated through the time gain compensation (TGC) device. Therefore, image uniformity is affected by the quality of TGC [32].

\subsection{Overall image quality}

The overall image quality depends on several properties like resolving capabilities, speckle pattern, image uniformity, size of the imaging area, penetration depth, size, and quality of the monitor. The overall image quality enhances the readability of an ultrasound image [32].

\section{SONOGRAPHIC ARTIFACTS THAT RESULT IN POOR QUALITY ULTRASOUND IMAGES}

Ultrasound images are affected by various artifacts that lead to poor quality of images. These artifacts are attributed to the following features of ultrasonography:

* The pulsed signals travel with a broad spectrum of frequency in a short duration, resulting in the creation of multiple noises in the image.

* The coherent nature of these signals increases the speckle noise of the images.

* Distortion of sound waves while traveling through tissues increases the incidence of artifacts in the images [33].

Artifacts may distort the size, shape, and position of the structures under examination. It may even show structures that are not present in the body [9]. Some of the artifacts are described below [Figure 10].

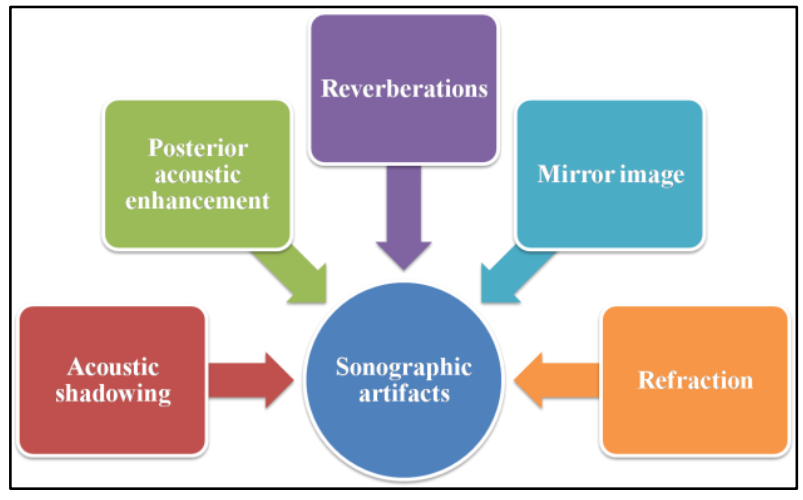

Figure 10: Types of ultrasonographic artifacts

The traditional screening method just looks for signs that a baby may be at an increased risk of having a chromosome abnormality, but not clear enough. Medical management of such abnormalities requires an organized approach of assessment, monitoring, and vigilance. In a birth defect condition where the vertebral column is open i.e. Spina bifida, common genetic disorders and congenital anomalies such as Down syndrome, beta-thalassemia, and neural tube defects are the issues related to drawbacks and consequences of poorquality ultrasound. Due to the absence of good quality and definite ultrasound guidelines and the additional lacunae in the awareness regarding the appropriate prenatal screening in the country, the optimum benefits of these screening protocols are not reaching the population.

\subsection{Acoustic shadowing}

Acoustic shadowing is caused due to the reduced intensity of echoes. This happens due to the presence of intervening structures with a greater attenuation coefficient than the structures deep within the body. In an ultrasound image, if a structure is brighter than other structures, it indicates strong reflection, likely high absorption, and weaker sound wave passage. Due to this, the other structure appears less echogenic than normal. Shadowing is commonly observed in bones and all types of calcifications [34].

\subsection{Posterior acoustic enhancement}

Posterior acoustic enhancement is the opposite of acoustic shadowing. This artifact is caused as a less attenuating structure is present at the surface resulting in stronger echoes, thereby, forming hyperechoic images of the underneath structures than the superficial structures. For instance, a blood vessel might be mistaken as a nerve in the presence of this artifact [35].

\subsection{Reverberations}

Reverberations are multiple reflections of an echo occurring when an ultrasound wave bounces between two or more reflectors, especially with high acoustic impedance. The ultrasonography machine recognizes these waves as equidistant parallel lines that gradually decreases the density of deeper lines. The image produced in the multiple representations of the same structure at different depths and is characterized by a striped pattern of alternating dark and clear lines at regular intervals $[36,37]$.

\subsection{Mirror artifacts}

Mirror artifact is a type of reverberation artifact, caused by the reflection of ultrasound waves at a specific angle. The waves are reflected by a strong reflective interface with high acoustic impedance. The resultant image will show an object similar to the true image on the opposite side of the structure. This 
image will be more distorted, hypoechoic, and blurred than the image of the original structure [37].

\subsection{Refraction}

Refraction also called a bayonet effect, is caused by a change in the direction of an ultrasound beam as it crosses the boundary of two regions with different speeds of sound. Refraction can cause errors in the lateral positioning of the internal structures [38].

\section{DEALING WITH ARTIFACTS}

The enhancement of ultrasound images primarily points towards adjusting controls on the ultrasound machine. It is classified into types: preprocessing techniques and postprocessing techniques. The former deals with image degradation due to the physical properties of the signals that include coherence, bandwidth, attenuation, absorption, etc.; while the latter uses signal processing techniques for the enhancement of the captured images, for instance, filtering and deconvolution [39-41]. Methods to enhance image quality are described below [Figure 11].
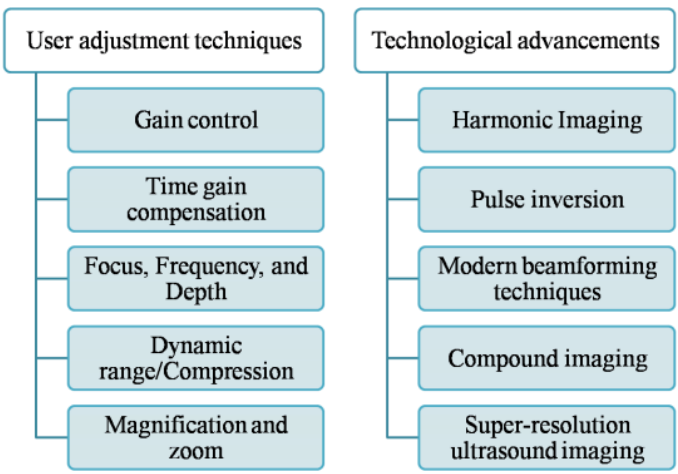

Figure 11: Methods to enhance ultrasound images

\subsection{User adjustment techniques}

\subsubsection{Gain control}

Gain refers to the strength of the signal. Gain control in the ultrasound machine increases the signal strength and brightness of the ultrasound image. Furthermore, it allows adjustment of the electronic amplification of echoes at all depths. The optimal gain necessary for image visualization might differ in various modes; thereby, requiring individual adjustments. It is also noted that increasing the gain can also affect lateral resolution $[3,9,18]$.

\subsubsection{Time gain compensation (TGC)}

TGC increases the signal strength which is restricted to a specified field of depth. We have seen earlier in the paper that attenuation increases with increasing depth. Therefore, to compensate for the attenuation, TGC allows a stepwise increase in gain that can be adjusted for a particular depth $[3,9]$.

\subsubsection{Focus, Frequency, and Depth}

When the ultrasound waves transverse through tissues, it converges to a point called the focal zone and then diverges. If the waves diverge beyond the focal zone, there will be a loss of information in a horizontal plane. Therefore, to minimize this loss, the focal zone should be adjusted at the same level as that of the target structure. Another aspect to improve an ultrasound image is the selection of frequency. Since these waves are more attenuated, a low-frequency probe is used for deeper structures, while a high-frequency probe for superficial structures. The depth control adjusts the depth of tissue that is displayed as the ultrasound image. The depth should be adjusted such that it demonstrates the tissues just deeper than the structures of interest. One should note that as the depth of the field increases, the quality of an image is compromised due to increased noise and automatic selection of low frequencies by modern transducers $[3,9,18]$.

\subsubsection{Dynamic range/Compression}

Dynamic range is defined as the ratio of the largest and the smallest signal levels in the image display. It is one of the processes to optimize the appearance of an ultrasound image on the display. The total dynamic range of an echo signal is too large and cannot be presented on a visual display. Therefore, the dynamic range of the detected echoes is compressed internally, thereby, resulting in a display with increased gain for smaller signal magnitudes and reduced gain for larger signal magnitudes.

\subsubsection{Magnification and zoom}

Magnification and zoom are two types of controls of image magnification. The former magnifies images resulting in degradation of the image resolution, while the latter magnifies images without degradation of the resolution. Though the image after magnification appears grainier than the unmagnified image, these are useful for making measurements [3].

\subsection{Technological advances to enhance image quality}

During the past decade, ultrasonography types of equipment have undergone several enhancements to improve imaging quality. Studies have demonstrated that resolution improves with an increase in frequency; therefore, it is important to increase the bandwidth or frequency of ultrasound to improve the resolution of the images [42-44]. However, the imaging depth decreases with increasing frequency. Recently, image processing techniques have been developed to improve image resolution without decreasing depth. These innovations are moving rapidly into widespread use, thereby, allowing the broader application of the modality in the medical field [45, 46]. Some of these advances are discussed below:

\subsubsection{Harmonic Imaging}

Ultrasound waves travel through the body in a linear and nonlinear fashion via tissues and microbubbles, respectively. When the waves travel in a distorted, nonlinear fashion, they generate harmonic components of the transmitted pulse inside the body. The initial harmonic wave inside the body is called the fundamental frequency or first harmonic. Harmonic imaging uses these components to generate an image. Furthermore, the second harmonic component is narrower and has a lower side-lobe artifact than the fundamental frequency in homogeneous beam propagation. These signals that are generated in the body have to pass only once through the fat layer, thereby, generating images with better spatial and contrast resolution $[47,48]$.

\subsubsection{Pulse inversion}

Pulse inversion is an application of harmonic imaging that maintains a balance between resolution and contrast. In this technique, an ultrasound beam, followed by its inverted replica, is transmitted through the body. Two echoes are detected that gives a nonzero value on subtraction in a nonlinear medium, while the sum of the echoes is equal to zero in a linear medium. Furthermore, pulse inversion technique can function on the entire bandwidth of the received signal, and as a result, improves resolution $[49,50]$. 


\subsubsection{Modern beamforming techniques}

The ultrasound beams generated by beamforming techniques are less affected by tissues in homogeneities. Therefore, they can improve wave penetration without sacrificing resolution and reduce artifacts like side-lobes and speed of sound errors. A few examples of modern beamforming techniques are dynamically focused reception and transmission, apodization, and reception, and pulse compression [51].

Dynamically focused reception is a technique that increases the focal length of the receiving beam by changing the time delays in such a way that the echoes from all depths within the tissue are always in focus. On the other hand, dynamically focused transmission forms an image by transmitting several pulse sequences focusing at different points. These techniques increase the depth of field by improving lateral resolution and reducing sidelobes [52]. A different approach to dynamic reception and transmission, called synthetic aperture, transmits a spherical wave from a single element in the transducer, thereby, creating a set of low-resolution images with the received signals. These images are combined and reconstructed into a high-resolution image. Therefore, the received images show improved resolution, contrast, and depth of field [53].

Apodization is a technique that applies weighing or shaping function across the receiving and transmitting apertures to reduce sidelobes and improve resolution. However, the typical apodization technique minimizes the sidelobes at the expense of the mainlobe lateral resolution. Therefore, several researchers have proposed theories to prevent this adverse outcome [54]. For example, in a study conducted by Guenther and Walker, the constrained least-squares theory was utilized to limit the energy of point spread function (PSF) within a certain area and achieve optimal contrast [55]. The pulse compression technique utilizes pulses with lower amplitude and longer duration as compared with the traditional pulse. The frequency of pulse compression is modulated with the aid of a binary code, or linear on nonlinear frequency modulation. It increases the signal-to-noise ratio that improves the penetration of pulses and providing images with good resolution. [56-59].

\subsubsection{Compound imaging}

Compound imaging is a technique to enhance ultrasound images by an approach that combines images using multiple frequencies, to reduce artifacts and improve resolution. In this technique, speckle is smoothed and averaged out making the images look less grainy. Recently, spatial compound imaging has been introduced in the ultrasound machine. In this mode, multiple ultrasound parallel beams are used to inspect the same tissue regions oriented along with different directions. Since multiple beams are used, the compound imaging frame rate is lower when compared with conventional B-mode imaging. Therefore, spatial compound images have reduced levels of artifacts including noise, clutter, speckle, and refractive shadows [47, 48]. Other compound imaging techniques o enhance images are frequency compounding and strain compounding.

Frequency compounding creates an ultrasound image either by dividing the spectrum of echoes into sub-bands on the receiving mode or by using multiple sources of waves at different frequencies at the transmitting end. Earlier, this technique worked on a fixed bandwidth system that compromised axial resolution to reduce speckle. To overcome this limitation, several studies have been conducted for increasing bandwidth. A study conducted by Sanchez et al. involved the use of resolution enhancement compression that combined frequency compounding with pulse compression. This doubled the bandwidth and axial resolution resulting in improved image contrast. Another scientist utilized a dual element transducer that operated at a high frequency of 20 and $40 \mathrm{MHz}$. This increased the sound-to-noise ratio of the image without compromising axial resolution. A technique using 2D directive filters was proposed by Dantas and Costa that applied these filters to the $2 \mathrm{D}$ radio frequency data. Speckle was reduced by compounding the output from these filters. In strain compounding, signals decorrelate in the presence of different strains created using external forces. These forces produce a three-dimensional tissue motion. By controlling the axial and lateral movements, speckle can be identified in the images as they move out of the plane motion in a different direction [60-63].

\subsubsection{Super-resolution ultrasound imaging}

Super-resolution is the capacity of an ultrasound scanner to distinguish between two objects or structures beyond the usual limit by using high-frequency acoustic waves. It utilizes a technique called ultrasound localization microscopy (ULM) to improve the resolution of ultrasound images. In this technique, a super-resolved composite image is formed through the accumulation of sub-wavelength localizations over the radiofrequency data or on the image. There are very few localization sources in each frame, therefore, their interference can be taken into account while visualizing the image [64-66].

\section{CONCLUSION}

Ultrasonography is a widely used imaging modality in the field of medical science. However, several artifacts in the ultrasound image result in poor imaging quality, thereby affecting the readability and interpretation of the results. Over the years, researchers have aimed to reduce the speckle noise and other artifacts present in ultrasound images. They have used various image processing techniques like harmonic imaging, modern beamforming techniques, and many more to enhance the image quality of ultrasound images. In the present paper, several user control adjustments are discussed that will help the user to manually adjust the settings of the ultrasound machine for better quality images. Furthermore, some of the recent technological advancements in the ultrasound system are covered to improve the understanding of these techniques for their use in clinical ultrasonography. In addition to enhanced image quality, these advances might aid in the transformation of medical ultrasonography into precision medicine to provide personalized patient care.

\section{ACKNOWLEDGMENTS}

The authors are thankful to Dr. Babasaheb Ambedkar Marathwada University for providing facilities to draft this paper.

\section{REFERENCES}

[1] Contreras Ortiz, S., Chiu, T. and Fox, M. 2012. Ultrasound image enhancement: A review. Biomedical Signal Processing and Control, 7(5), 419-428.

[2] Hangiandreou, N. 2003. AAPM/RSNA Physics Tutorial for Residents: Topics in US. RadioGraphics, 23(4), 1019-1033.

[3] Thoirs, K. 2012. Physical and technical principles of sonography: A practical guide for non- 
sonographers. Radiographer, 59(4), 124-132.

[4] Chan, V. and Perlas, A. 2010. Basics of Ultrasound Imaging. In Atlas of Ultrasound-Guided Procedures in Interventional Pain Management. Springer Science+Business Media, 13-19.

[5] Bercovich, E. and Javitt, M. 2018. Medical Imaging: From Roentgen to the Digital Revolution, and Beyond. Rambam Maimonides Med J, 9(4), e0034.

[6] Kaproth-Joslin, K., Nicola, R. and Dogra, V. 2015. The History of US: From Bats and Boats to the Bedside and Beyond:RSNA Centennial Article. RadioGraphics, 35(3), 960-970.

[7] Cronan, J. 2006. Ultrasound: Is There a Future in Diagnostic Imaging? J Am Coll Radiol, 3(9), 645-646.

[8] Moreau, J. 2007. Re: "Ultrasound: Is There a Future in Diagnostic Imaging?”. J Am Coll Radiol, 4(1), 78-79.

[9] Shanthanna, H. 2014. Review of essential understanding of ultrasound physics and equipment operation. World J Anesthesiol, 3(1), 12-17.

[10] Cootney, R. 2001. Ultrasound Imaging: Principles and Applications in Rodent Research. ILAR Journal, 42(3), 233-247.

[11] Abu-Zidan, F., Hefny, A. and Corr, P. 2011. Clinical ultrasound physics. J Emerg Trauma Shock, 4(4), 501503.

[12] Wells, P. 1998. Physics and bioeffects. In Diagnostic Ultrasound, A logical approach. Philadelphia: LppincottRaven Publishers, 1-19.

[13] Tole, N. 2005. Basic physics of ultrasonographic imaging. WHO Library Cataloguing-in-Publication Data, $1-95$.

[14] Sites, B., Brull, R., Chan, V., Spence, B., Gallagher, J., Beach, M., et al. 2007. Artifacts and pitfall errors associated with ultrasound-guided regional anesthesia. Part I: understanding the basic principles of ultrasound physics and machine operations. Reg Anesth Pain Med, 32(5), 412-418.

[15] Rose, J. and Bair, A. 2006. Fundamentals of ultrasound. In Practical guide to Emergency Ultrasound. PA: Lippincott Williams and Wilkins, 27-41.

[16] Schuler, A. 2008. Image artifacts and pitfalls. In Chest sonography. 2nd ed. New York: Springer, 175-182.

[17] Abuhamad, A. 2014. Basic physical principles of medical ultrasound. In Ultrasound in Obstetrics and Gynecology, 9-29.

[18] Abuhamad, A. 2014. Basic Characteristics of the Ultrasound Equipment. In Ultrasound in Obstetrics and Gynecology, 30-42.

[19] Kossoff, G. 2000. Basic Physics and Imaging Characteristics of Ultrasound. World J Surg, 24(2), 134142.

[20] Soler López, F., Mayorga Betancour, M. and Cruz Salazar, E. 2013. Application of ultrasound in medicine Part II: the ultrasonic transducer and its associated electronics. Tecciencia, 8(15), 14-26.

[21] Enriquez, J. and $\mathrm{Wu}, \mathrm{T}$. 2014. An introduction to ultrasound equipment and knobology. Crit. Care Clin,
30(1), 25-45.

[22] Najafi, T. and Sepehri N. 2008. A novel hand-controller for remote ultrasound imaging. Mechatronics, 18(10), $578-590$.

[23] Magud, O., Tuba, E. and Bacanin, N. 2016. An Algorithm for Medical Ultrasound Image Enhancement by Speckle Noise Reduction. Int. J. Signal Process, 1, 146-151.

[24] Carovac, A., Smajlovic, F. and Junuzovic, D. 2011. Application of Ultrasound in Medicine. Acta Inform Med, 19(3), 168-171.

[25] Moorthy, R. 2002. Doppler ultrasound. Med J Armed Forces India, 58(1), 1-2.

[26] Ng, A. and Swanevelder, J. 2011. Resolution in ultrasound imaging. Contin Educ Anaesth Crit Care Pain, 11(5), 186-192.

[27] Ng, A. and Swanevelder, J. 2010. Perioperative monitoring of left ventricular function: what is the role of recent developments in echocardiography? Br J Anaesth, 104, 669-672.

[28] Ng, A. and Swanevelder, J. 2009. Perioperative echocardiography for non-cardiac surgery: what is its role in routine haemodynamic monitoring? $\mathrm{Br} \mathrm{J}$ Anaesth, $102,731-734$.

[29] Marhofer, P., Harrop-Griffiths, W., Willschke, H. and Kirchmair, L. 2010. Fifteen years of ultrasound in regional anaesthesia: part 2. Recent developments in block techniques. Br J Anaesth, 104, 673-683.

[30] Fiegenbaum, H. 1994. Echocardiography. 5th ed. Philadelphia: Lea \&Febiger, 1.

[31] Lieu, D. 2010. Ultrasound Physics and Instrumentation for Pathologists. Arch Pathol Lab Med, 134(10), 15411556.

[32] Alasaarela, E. and Koivukangas, J. 1990. Evaluation of Image Quality of Ultrasound Scanners in Medical Diagnostics. J Ultrasound Med, 9, 23-34.

[33] Arturo, F. and López, S. 2013. Ultrasound applications to medicine part 1: physical principles. Tecciencia, 7(14), 77-89.

[34] Kremkau, F. and Taylor, K. 1986. Artifacts in ultrasound imaging. J Ultrasound Med, 5, 227-237.

[35] Sites, B., Brull, R., Chan, V., Spence, B., et. al. 2007. Artifacts and pitfall errors associated with ultrasoundguided regional anesthesia. Part II: a pictorial approach to understanding and avoidance. Reg Anesth Pain Med, 32, 419-433.

[36] Thickman, D., Ziskin, M., Goldenberg, N. and Linder, B. 1983. Clinical manifestations of the comet tail artifact. J Ultrasound Med, 2, 225-230.

[37] Feldman, M., Katyal, S. and Blackwood, M. 2009. US artifacts. Radiographics, 29(4), 1179-1189.

[38] Gray, A. and Schafhalter-Zoppoth, I. 2005. "Bayonet artifact" during ultrasound-guided transarterial axillary block. Anesthesiology, 102, 1291-1292.

[39] Ploquin, M., Basarab, A. and Kouamé, D. 2015. Resolution enhancement in medical ultrasound imaging. J Med Imaging (Bellingham), 2(1), 017001. 
[40] Tortoli, P. and Jensen, J. 2006. Introduction to the Special Issue on Novel Equipment for Ultrasound Research. IEEE Transactions on Ultrasonics, Ferroelectrics, and Frequency Control, 53(10), 17051706.

[41] Clement, G., Huttunen, J. and Hynynen, K. 2005. Superresolution ultrasound imaging using back-projected reconstruction. J Acoust Soc Am, 118(6), 3953-3960.

[42] Foster, F., Pavlin, C., Harasiewicz, K., Christopher, D. and Turnbull, D. 2000. Advances in ultrasound biomicroscopy. Ultrasound Med Biol, 26(1), 1-27.

[43] Blomgren, P., Papanicolaou, G. and Zhao, H. 2002. Super-resolution in time-reversal acoustics. J Acoust Soc Am, 111(1 Pt 1), 230-248.

[44] Jirík, R. and Taxt, T. 2006. High-resolution ultrasonic imaging using fast two-dimensional homomorphic filtering. IEEE Trans UltrasonFerroelectr Freq Control, 53(8), 1440-1448.

[45] van Sloun, R., Cohen, R. and Eldar, Y. 2020. Deep Learning in Ultrasound Imaging. Proceedings of the IEEE, 108(1), 11-29.

[46] Clement, G., Huttunen, J. and Hynynen, K. 2005. Superresolution ultrasound imaging using back-projected reconstruction. J Acoust Soc Am, 118(6), 3953-3960.

[47] Jespersen, S., Wilhjelm, J. and Sillesen, H. 1998. Multiangle compound imaging. Ultrason Imaging, 20, 81102 .

[48] Weng, L., Tirumalai, A., Lowery, C., et al. 1997. US extended-field-of-view imaging technology. Radiology, 203, 877-880.

[49] Simpson, D., Chien Ting Chin, and Burns, P. 1999. Pulse inversion Doppler: a new method for detecting nonlinear echoes from microbubble contrast agents. IEEE Transactions on Ultrasonics, Ferroelectrics, and Frequency Control, 46(2), 372-382.

[50] Frinking, P., Bouakaz, A., Kirkhorn, J., Ten Cate, F. and de Jong, N. 2000. Ultrasound contrast imaging: current and new potential methods. Ultrasound Med Biol, 26(6), 965-975.

[51] Kollmann, C. 2007. New sonographic techniques for harmonic imaging-Underlying physical principles. European Journal of Radiology, 64(2), 164-172.

[52] Lu, J-Y., Zou, H., and Greenleaf, J. 1994. Biomedical ultrasound beam forming. Ultrasound in Medicine \& Biology, 20(5), 403-428.

[53] Jensen, J., Nikolov, S., Gammelmark, K. and Pedersen, M. 2006. Synthetic aperture ultrasound imaging. Ultrasonics, 44 (Suppl 1), e5-15.

[54] Seo, C. and Yen, J. 2008. Sidelobe Suppression in
Ultrasound Imaging using Dual Apodization with Crosscorrelation. IEEE Trans Ultrason Ferroelectr Freq Control, 55(10), 2198-2210.

[55] Guenther, D. and Walker, W. 2007. Optimal apodization design for medical ultrasound using constrained least squares part II simulation results. IEEE Transactions on Ultrasonics, Ferroelectrics, and Frequency Control, 54(2), 343-358.

[56] Chiao, R. and Hao, X. 2005. Coded excitation for diagnostic ultrasound: a system developer's perspective. IEEE Trans Ultrason Ferroelectr Freq Control, 52(2), 160-170.

[57] Misaridis, T. and Jensen, J. 2005. Use of modulated excitation signals in medical ultrasound. Part I: basic concepts and expected benefits. IEEE Trans Ultrason, Ferroelect, Freq Contr, 52(2), 177-191.

[58] Misaridis, T. and Jensen, J. 2005. Use of modulated excitation signals in medical ultrasound. Part II: design and performance for medical imaging applications. IEEE Trans Ultrason, Ferroelect, Freq Contr, 52(2), 192-207.

[59] Misaridis, T. and Jensen, J. 2005. Use of modulated excitation signals in medical ultrasound. Part III: high frame rate imaging. IEEE Trans Ultrason, Ferroelect, Freq Contr, 52(2), 208-219.

[60] Chang, J., Kim, H., Lee, J. and Shung, K. 2010 Frequency Compounded Imaging with a High-Frequency Dual Element Transducer. Ultrasonics, 50(0), 453-457.

[61] Dantas, R. and Costa, E. 2007. Ultrasound speckle reduction using modified gabor filters. IEEE Trans Ultrason, Ferroelect, Freq Contr, 54(3), 530-538.

[62] Sanchez, J. and Oelze, M. 2009. An ultrasonic imaging speckle-suppression and contrast-enhancement technique by means of frequency compounding and coded excitation. IEEE Trans Ultrason, Ferroelect, Freq Contr, 56(7), 1327-1339.

[63] Pc, L. and Mj, C. 2002. Strain compounding: a new approach for speckle reduction. IEEE Trans Ultrason Ferroelectr Freq Control, 49(1), 39-46.

[64] Christensen-Jeffries, K., Couture, O., Dayton, P., Eldar, Y., Hynynen, K., Kiessling, F., et al. 2020. Superresolution Ultrasound Imaging. Ultrasound in Medicine and Biology, 46(4), 865-891.

[65] Couture, O., Hingot, V., Heiles, B., Muleki-Seya, P. and Tanter, M. 2018. Ultrasound Localization Microscopy and Super-Resolution: A State of the Art. IEEE Trans Ultrason Ferroelectr Freq Control, 65(8), 1304-1320.

[66] Desailly, Y., Pierre, J., Couture, O. and Tanter, M. 2015. Resolution limits of ultrafast ultrasound localization microscopy. Phys Med Biol, 60(22), 8723-8740. 